\title{
Der FOKUS-Ansatz: Hyperaktive, impulsive und unaufmerksame Kinder nach der Einschulung fördern
}

Sara Benini, Jennifer C. Fräulin und Markus P. Neuenschwander

\section{Zusammenfassung}

Unaufmerksamkeit, Hyperaktivität und Impulsivität sind frühe Indikatoren für einen gefährdeten Bildungsverlauf. Zur Förderung von Kindern mit diesen Verhaltensweisen im Grundschulunterricht wurde der FOKUS-Ansatz entwickelt. Der FOKUS-Ansatz enthält praktische pädagogische Massnahmen gegenüber Kindern mit einer Neigung zur ADHS Symptomatik im Unterricht - in Ergänzung zu allfälligen Medikamenten. Es wurden konkrete Elemente (1) einer wirksamen Klassenführung (Klassenebene), (2) des Umgangs mit einzelnen unaufmerksamen, hyperaktiven und impulsiven Kindern (individuelle Ebene) und (3) der Zusammenarbeit mit den Eltern dieser Kinder (Elternebene) wissenschaftsbasiert erarbeitet und in Zusammenarbeit mit Praktikerinnen konkretisiert. Zur Vermittlung des FOKUS-Ansatzes wurde eine Weiterbildung mit rund 90 Lehrpersonen durchgeführt. Eine Evaluation im Vergleich mit einer Kontrollgruppe zeigte, dass die Lehrpersonen wichtige Weiterbildungsinhalte umsetzten und dass dies zur Zunahme der Aufmerksamkeit und der sozialen Integration der Kinder in die Klasse geführt hat. Der Effekt auf die Hyperaktivität/Impulsivität war nur ansatzweise zu finden. Der FOKUS-Ansatz und die Weiterbildung haben sich als erfolgreich erwiesen und werden zukünftig für Lehrpersonen angeboten.

\section{Schlagworte}

Hyperaktivität/Impulsivität, Unaufmerksamkeit, Schule, Weiterbildung, Wirkungsstudie 


\section{$1 \quad$ Einleitung}

Mit der Einschulung beginnt für die Kinder ein neuer Lebensabschnitt, welcher Herausforderungen mit sich bringt. Dazu gehören unter anderem die Positionierung innerhalb des Klassenverbands, das Kennenlernen der Lehrperson/en sowie veränderte Tagesstrukturen. Dabei zeigt sich, dass manche Kinder grosse Mühe haben, lange still zu sitzen und konzentriert Aufgaben zu bearbeiten. Treten diese Verhaltensweisen besonders ausgeprägt und häufig auf, kann es nach gründlicher klinischer Abklärung zur Diagnostizierung einer Aufmerksamkeitsdefizit-Hyperaktivitätsstörung (ADHS) kommen. ADHS wird nach dem US-Amerikanischen Klassifikationsschema DSM-5 (APA, 2013) in zwei Kernsymptome unterteilt: a) Hyperaktivität/Impulsivität und b) Unaufmerksamkeit. Es wird beobachtet, dass hohe Ausprägungen von Hyperaktivität/Impulsivität und Unaufmerksamkeit ohne Behandlung ein Risiko für die zukünftige gesundheitliche sowie soziale und schulische Entwicklung der Betroffenen darstellen können. Hyperaktives/ impulsives Verhalten äussert sich anhand exzessiver Ruhelosigkeit sowie anhand von Ungeduld und Schwierigkeiten, etwas abwarten und Bedürfnisse aufschieben zu können. Unaufmerksames Verhalten in der Schule äussert sich anhand von häufigen, vorzeitigen Abbrüchen von Aktivitäten und unordentlicher, nachlässiger Arbeitsweise. Hyperaktivität/Impulsivität sowie Unaufmerksamkeit wirken sich in vielfältiger Art negativ auf die Situation in der Schule, das Lernen, die Leistungen und die Schulkarriere der Kinder aus (Barkley, 2006, Döpfner, 2001). Verhaltensauffällige Kinder zeigen tiefere Leistungen als ihre Mitschülerinnen und Mitschüler, obwohl sie aufgrund ihrer kognitiven Fähigkeiten im Unterricht durchschnittliche Leistungen erbringen könnten (Döpfner, Frölich \& Lehmkuhl, 2013). Weiter zeigte eine Studie, dass Kinder und Jugendliche mit ADHS häufiger als ihre Mitschülerinnen und Mitschüler Schultypen mit Grundanforderungen oder kürzere Berufsausbildungen besuchen (Bader, Pierrehumbert, Junier \& Halfon, 2005). Neuere Untersuchungen konnten direkte Auswirkungen von Verhaltensproblemen im Unterricht auf die Übertrittsentscheidungen in die Sekundarstufe I nachweisen (Neuenschwander \& Malti, 2009). Langzeitstudien weisen zudem darauf hin, dass verhaltensauffällige und unaufmerksame Kinder ein höheres Risiko haben, in Sonderschulen platziert zu werden. Mit grösserer Wahrscheinlichkeit riskieren sie ihre Ausbildung vorzeitig abzurechen oder Berufsausbildungen mit niedrigem Anforderungsniveau aufzunehmen (Barkley, Fischer, Edelbrock \& Smallish, 1991; Mannuzza, Klein, Bessler, Malloy \& Hynes, 1997).

Um den genannten langfristigen Beeinträchtigungen für den Schulverlauf vorzubeugen, sollte eine Intervention wegen der Situationsspezifität der Symptomatik und ihren vielfältigen Ausprägungen dort ansetzen, wo die Probleme auftreten 
(Döpfner, Schürman \& Frölich, 2002). Es sind demzufolge effektive Massnahmen erforderlich, die im Unterrichtsalltag einen nachgewiesenen positiven Effekt auf Verhaltensauffälligkeit und Unaufmerksamkeit haben. Diese Massnahmen sind auch bei einer klinisch nicht diagnostizierten ADHS notwendig und sollten von Lehrpersonen in der Regelklasse umgesetzt werden können. Es stellt sich somit die Frage, wie Kinder mit Neigung zu Unaufmerksamkeit und Hyperaktivität/Impulsivität im Unterricht nach der Einschulung besonders gefördert werden können.

\section{Der FOKUS-Ansatz}

Vor dem dargestellten Hintergrund wurde ein Konzept zu einem angemessenen Umgang der Lehrpersonen mit Schülerinnen und Schülern mit Verhaltens- und Aufmerksamkeitsproblemen im Unterricht und deren Eltern entwickelt, der FOKUS-Ansatz ${ }^{1}$ (Förderung von Kindern mit Verhaltensauffälligkeit und Unaufmerksamkeit in der Schule). In Abbildung 1 sind die dem FOKUS-Ansatz zugrunde gelegten Annahmen graphisch dargestellt.

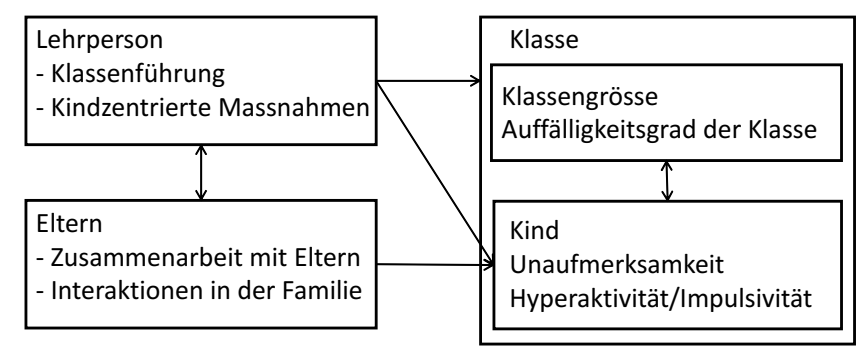

Abb. 1 Arbeitsmodell des FOKUS-Ansatzes

Es wird davon ausgegangen, dass Lehrpersonen durch eine sensibilisierte Einstellung und geeignete Handlungen die aufkommenden Schwierigkeiten erfolgreich und

1 Für eine detaillierte Beschreibung des FOKUS-Ansatzes und der Weiterbildung s. Neuenschwander, M. P. \& Benini, S. (2016). FOKUS - Förderung von Kindern mit Verhaltensauffälligkeiten und Unaufmerksamkeit im Unterricht. Schlussbericht z.Hd. des Bundesamtes für Gesundheit. Solothurn: Zentrum Lernen und Sozialisation der PH FHNW (www.fhnw.ch/ph/zls/fokus) 
nachhaltig bearbeiten und dadurch Schülerinnen und Schüler in ihrer schulischen Entwicklung fördern können. Auf der Ebene der Klassenführung sind eine hohe Strukturierung der Lerntätigkeit und des Lerngegenstandes (Klassenebene) sowie eine möglichst individuelle Anleitung des Kindes (individuelle Ebene) notwendig (Frölich, Döpfner \& Banaschewski, 2014; Zentall, 1993; Gawrilow, Guderjahn \& Gold, 2013). Um diesen Kindern eine erfolgreiche Mitarbeit in der Klassengemeinschaft zu ermöglichen, benötigt es seitens der Lehrpersonen einen strukturierenden und wertschätzenden Umgang. Damit kann auch bei den Mitschülerinnen und Mitschülern Verständnis und Akzeptanz geweckt werden.

Neben den Massnahmen, welche eine Lehrperson auf Klassenniveau umsetzen kann, sind gezielte, kindspezifische Massnahmen im Unterricht hilfreich. Es handelt sich um Massnahmen zur Aufmerksamkeitsförderung oder zur Förderung der Selbstregulation, welche den Kindern beim Denken, Planen und Handeln helfen. Das Ziel ist es, den Kindern zu helfen, ihre grundsätzlich vorhandenen Fähigkeiten in der richtigen Situation zum richtigen Zeitpunkt in Verhalten umzusetzen (Hoberg, 2013).

Darüber hinaus nimmt auch die Zusammenarbeit mit den Eltern der Schülerinnen und Schüler einen grossen Stellenwert ein (DuPaul et al., 2011; Mautone, Lefler \& Power, 2011). Eine gelingende Zusammenarbeit basiert auf drei Prinzipien: Austausch von Informationen, Aufbau von Vertrauen und Koordination von Massnahmen (Neuenschwander et al., 2005).

\subsection{Konzeption der Weiterbildung}

Ausgehend vom FOKUS-Ansatz wurde eine Weiterbildung zu folgenden drei Elementen der pädagogischen Förderung verhaltensauffälliger und unaufmerksamer Kinder im Unterricht erarbeitet (Neuenschwander \& Benini, 2017/im Druck): a) klassenzentrierte Massnahmen (Klassenführung), b) kinderzentrierte Massnahmen, insbesondere die Förderung der Selbstregulation, und c) förderliche Zusammenarbeit mit den Eltern. Die Weiterbildung richtet sich an Lehrpersonen der Unterstufe (1. und 2. Klasse). In Abbildung 2 ist die inhaltliche Struktur der FOKUS-Weiterbildung zusammenfassend dargestellt. Bei der Entwicklung der Weiterbildung wird das Schwergewicht auf wissenschaftlich überprüfte und wirksame pädagogische Strategien zur Förderung der Kinder gelegt, welche sinnvoll kombiniert werden, so dass sich am ehesten ein Erfolg für die Lehrpersonen und die Kinder einstellt. 


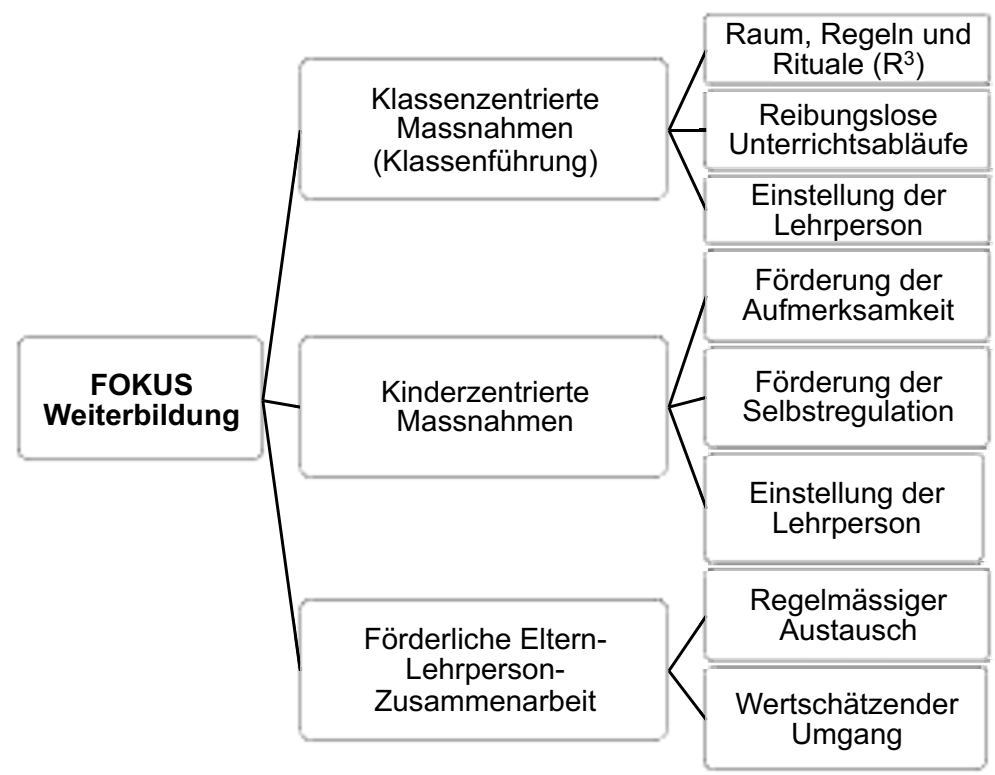

Abb. 2 Inhaltliche Struktur der FOKUS-Weiterbildung

Die Weiterbildung für Lehrpersonen ist so aufgebaut, dass zu jedem der drei Elemente des FOKUS-Ansatzes jeweils ein kurzer Theorieinput gegeben und eine themenspezifische Praxis-Checkliste verteilt wird. Danach werden konkrete Anwendungsbeispiele und Materialien im Plenum oder innerhalb kleiner Gruppen eingeführt und bearbeitet. Für die Wirksamkeit der Weiterbildung ist es wesentlich, dass Lehrpersonen die Elemente der Weiterbildung selbstständig und ohne grossen Aufwand im Unterricht einsetzen können (Gawrilow et al., 2013). Die Weiterbildung ist deshalb so konzipiert, dass sie sich über einen längeren Zeitraum erstreckt und dass die Lehrpersonen zwischen den Schulungen dank der zeitlichen Abstände (mind. ein Monat) immer die Möglichkeit zur Erprobung der Fortbildungsinhalte haben.

\subsection{Klassenzentrierte Massnahmen}

Lehrpersonen unterrichten erfolgreich, wenn sie klare Verhaltenserwartungen an die Kinder formulieren und ihnen Strukturierungshilfen bereitstellen (Jarman, 1996, Kos, Richdale \& Hay, 2006). Ein wesentlicher Aspekt dieses Teils der Wei- 
terbildung ist somit die Anregung der Selbstreflexion zur eigenen Klassenführung und zur Qualität des Unterrichts. Als Mittel zur "wertschätzenden Strukturierung“ des Unterrichts wird das Element Raum-Regeln-Rituale (R3) entwickelt und in die Weiterbildung eingeführt. Dabei stehen präventiv-proaktive Massnahmen im Vordergrund. In Bezug auf die Raumstrukturierung wird insbesondere darauf geachtet, dass im Klassenzimmer allfällige Reizüberflutungen auf ein Minimum reduziert werden. Bereits ab den ersten Wochen nach der Einschulung spielen bei der Strukturierung des Unterrichtsgeschehens, geeignete Klassenregeln und ritualisierte Unterrichtsabfolgen ebenfalls eine wichtige Rolle. Die Lehrpersonen werden im Rahmen der Weiterbildung auch für die selbsterfüllende Wirkung von Erwartungen sensibilisiert. Mittels Beispielen erfolgreicher Bildungsverläufe verhaltensauffälliger Kinder, akkurater Informationen zu Verhaltensauffälligkeiten und Unaufmerksamkeit im Kindesalter und Hinweisen sowie Erläuterungen zur Wirkung der Lehrpersoneneinstellung auf die Kinder wird das Bewusstsein der Lehrpersonen für diese Angelegenheit erweitert und verstärkt.

\subsection{Kinderzentrierte Massnahmen}

Zusätzlich zu allgemeinen Massnahmen sind individuelle, spezifische Interventionen beim Kind notwendig (DuPaul \& Stoner, 2003). Individuelle Massnahmen haben den Vorteil, dass sie auf die spezifische Situation des Kindes abgestimmt werden können. Sie setzen aber voraus, dass individualisiert unterrichtet wird. Bewegung gehört im Kindesalter zu einem gesunden Alltag. Den Lehrpersonen werden unterschiedliche Möglichkeiten vorgestellt, wie sie koordinative Bewegungsaufgaben bei Lern- und Wahrnehmungsvorgängen einbauen können. Weil das aufmerksame Zuhören eine der grundlegenden Aufgaben der Schülerinnen und Schüler in der Schule ist und Anleitungen von Arbeitsaufträgen im Unterricht in der Regel mündlich (d.h. akustisch) erteilt werden, wird den Lehrpersonen zur Aufmerksamkeitsförderung auch ein vom Projektteam selbst entwickeltes akustisches Konzentrationstraining (Ein FOKUS auf Ohr) vorgestellt. Die Lehrpersonen führen die Übungen zwei bis drei Mal in der Woche mit dem hyperaktiven/impulsiven und/oder unaufmerksamen Kind durch. Zur erfolgreichen Förderung und Unterstützung der Selbstregulation der Kinder bieten Wenn-Dann-Pläne eine effektive Lösung (Gawrilow, Schmitt \& Rauch, 2011; Gawrilow, Gollwitzer \& Oettingen, 2011). Den Lehrpersonen werden konkrete Beispiele und Materialien präsentiert, wie Wenn-Dann-Pläne erfolgreich im Unterricht eingesetzt werden können. 


\subsection{Förderliche Eltern-Lehrperson-Zusammenarbeit}

Um einen regelmässigen Kontakt mit den Eltern des Schülers oder der Schülerin zu pflegen, wird den Lehrpersonen die Wochenkarte vorgestellt, welche im $\mathrm{Zu}$ sammenhang mit der Durchführung der Wenn-Dann-Pläne eingesetzt werden kann. Die Lehrperson notiert auf der Karte, welcher Wenn-Dann-Plan das Kind gerade durchführt und wie die Umsetzung während der Woche gelaufen ist. Zum wertschätzenden Umgang mit dem verhaltensauffälligen Kind notiert die Lehrperson immer auch ein Lob der Woche. Zusätzlich werden die Lehrpersonen in das ElternInfoBlatt eingeführt. Dabei handelt es sich um ein Instrument zur Kommunikation einer ausführlichen Situationsbestimmung über das kindliche Verhalten in der Klasse, welches die Lehrpersonen drei bis vier Mal im Jahr ausfüllen und mit den Eltern persönlich besprechen. Schliesslich werden den Lehrpersonen gezielte Techniken der förderlichen Gesprächsführung mit Eltern vorgestellt und mit ihnen im Rahmen der Schulung geübt.

\subsection{Fragestellungen}

Vor diesem Hintergrund wurde in der vorliegenden Studie die Bearbeitung von Verhaltens- und Aufmerksamkeitsproblemen in der Schule untersucht. Die folgenden Forschungsfragen wurden verfolgt: a) Wie können Lehrpersonen im Hinblick auf die Optimierung ihrer Klassenführung effektiv weitergebildet werden? b) Wie verändert sich der Umgang der Lehrperson mit verhaltensauffälligen Kindern aufgrund der Weiterbildung? c) Können Unaufmerksamkeit und impulsive sowie hyperaktive Verhaltensweisen von Kindern des 2. Schuljahres durch eine konzeptbasierte Klassenführung, ergänzt mit kindspezifischen Massnahmen, reduziert werden?

Es wird demzufolge geprüft, ob eine eigens dafür entwickelte Intervention in Form einer Lehrpersonenweiterbildung einen positiven Effekt auf die Förderung von Verhaltensauffälligkeiten und Unaufmerksamkeit im Unterricht hat. Aus Platzgründen wird auf das Element der Elternzusammenarbeit im Folgenden nicht näher eingegangen. 


\section{$3 \quad$ Methode}

\subsection{Teilnehmende Lehrpersonen (Stichprobe)}

Die Stichprobe setzte sich aus Volksschullehrpersonen der 1.-2. Klasse aus neun Deutschschweizer Kantonen (67.9\% der Schulen in ländlichen Gebieten) zusammen. Das Durchschnittsalter der Lehrpersonen ( $94.9 \%$ weiblich) betrug $M=42.36$ Jahre $(S D=12.39)$ bei einer durchschnittlichen Berufserfahrung von $M=15.92$ Jahren $(S D=10.85)$. Aus jeder Klasse wurde jenes Kind in die Stichprobe miteinbezogen, dessen Eltern einer Teilnahme an der Studie zugestimmt hatten und welches gemäss dem ADHS-Index die höchste Ausprägung des Auffälligkeitswerts aufwies (Kapitel 3.3.1). Die teilnehmenden Kinder (79.6\% männlich) waren im Durchschnitt $M=8.65$ Jahre alt $(S D=1.02)$. Von den 137 Lehrpersonen, welche an der ersten Situationsanalyse (Pretest) teilgenommen hatten, waren an der zweiten Situationsanalyse (Posttest) noch 133 Lehrpersonen bei der Studie dabei. Insgesamt wurden die Angaben von vier Lehrpersonen aus den Analysen ausgeschlossen, weil sie im Pre- und/oder im Posttest den höchsten Belastungswert aufwiesen. Die Messwerte dieser Lehrpersonen wurden als sogenannte Ausreisser behandelt, welche zugunsten der Verlässlichkeit der Ergebnisse nicht in die Analysen einbezogen wurden. Wie frühere Studien zeigten, kann eine sehr hohe Belastung nämlich zu einer verzerrten Beurteilung des Schülerverhaltens führen. So beschrieben zum Beispiel Maslach und Jackson (1981) das Burnout-Syndrom bei stark belasteten Lehrpersonen unter anderem mit einer veränderten Schülerwahrnehmung (vgl. auch Heim \& Nido, 2008). Die Lehrpersonen und ihre Klassen wurden in drei Gruppen (Gruppe A, Gruppe AB und Kontrollgruppe) aufgeteilt. Diese unterschieden sich nicht signifikant in Bezug auf folgende Merkmale: Geschlecht, Durchschnittsalter der Lehrpersonen, durchschnittliche Berufserfahrung, Klassengrösse, Anteil Schülerinnen und Schüler mit Migrationshintergrund sowie Auffälligkeitswert des in die Studie einbezogenen Kindes.

\subsection{Durchführung}

Die Lehrpersonen wurden postalisch über die Schulleitung um eine Teilnahme an der Studie angefragt. Nachdem für jede teilnehmende Lehrperson die unterschriebenen Elterneinverständniserklärungen der Schülerinnen und Schüler vorlagen, wurde das Zielkind ausgewählt (Kapitel 2.3.1). Es fanden zwei Situationsanalysen statt. Die erste (Pretest) fand zwischen April und Juni 2014, die zweite (Posttest) zwischen Januar und April 2015 statt. Dabei wurde das Zielkind jeweils während 
einer Schullektion im Unterricht beobachtet, und die Lehrperson füllte einen Onlinefragebogen aus. Aufgrund des quasi-experimentellen Designs wurden die Lehrpersonen in drei Gruppen aufgeteilt: zwei Experimentalgruppen (A und $\mathrm{AB})$ und eine Kontrollgruppe. Lehrpersonen der Gruppe A wurden lediglich in den klassenzentrierten Massnahmen weitergebildet, während die Lehrpersonen der Gruppe AB zusätzlich zu kinderzentrierten Massnahmen und förderlicher Eltern-Lehrperson-Zusammenarbeit weitergebildet wurden (Kapitel 1.1). Lehrpersonen der Kontrollgruppe besuchten hingegen keine Weiterbildung. Zwischen den Situationsanalysen nahmen die Lehrpersonen der Experimentalgruppen im Juni, November und Dezember 2014 an den FOKUS-Weiterbildungen teil. Im September erhielten sie ein individuelles Coaching durch eine der beiden Weiterbildnerinnen.

\subsection{Instrumente}

\subsubsection{ADHS-Index}

In dieser Studie wurde nicht klinisch gearbeitet. Bei der Beurteilung des kindlichen Verhaltens hat man sich jedoch an den Dimensionen von ADHS orientiert. Die Lehrpersonen wurden gebeten für jedes Kind dessen Hyperaktivität, Impulsivität und Unaufmerksamkeit einzuschätzen. Zur Einschätzung verwendeten sie ein dafür vorgesehenen Instrument. Jede Lehrperson füllte somit für ihre Klasse einen ADHS-Index für Lehrpersonen (Lidzba, Christiansen, Drechsler, 2013) aus. Der Index besteht aus 10 Aussagen zu den Verhaltensweisen des Kindes innerhalb des letzten Monates (z. B. «Zappelt oder rutscht auf dem Stuhl herum»). Die Lehrperson sollte dabei angeben, wie gut diese Aussagen den jeweiligen Schüler/die jeweilige Schülerin beschreiben ( 0 ,traf überhaupt nicht auf das Kind zu' bis 3 ,traf ganz genau auf das Kind zu'). Für die Auswertung wurden die Antworten aufsummiert und in Abhängigkeit von Alter und Geschlecht ein Auffälligkeitswert (1, nicht auffällig' bis 4 ,sehr auffällig') bestimmt (Pretest: $M=3.69$ Punkte; $S D=.73$; Posttest: $M=2.55$ Punkte; $S D=1.10$ ). In jeder Klasse wurde jenes Kind mit dem höchsten Auffälligkeitswert in die Studie aufgenommen.

\subsubsection{Verhaltensbeobachtung}

Jedes Kind wurde während einer Lektion von einem Projektmitarbeitenden beobachtet. Dabei wurden mittels eines Beobachtungsrasters das methodisch didaktische Setting, die Verhaltensweisen des Kindes sowie die Lehrpersonenreaktionen darauf pro Minute festgehalten. Den geschulten Beobachtenden lag dabei eine standardisierte, schriftliche Instruktion vor. Eine Audiodatei, welche über einen Kopfhörer 
abgehört wurde, diente der Zeiteinteilung. Zudem sassen die Beobachtenden so, dass sie das Kind frontal oder seitlich sehen konnten. Für jede Beobachtungseinheit (1 Minute) wurden das Verhalten des Kindes anhand von vier Unterkategorien (,Regelverhalten', ,Unaufmerksamkeit', ,Hyperaktivität' und ,Impulsivität') kodiert. Gleichzeitig wurde die Lehrpersonenreaktion (,Lehrperson (LP) reagiert nicht auf das Verhalten des Kindes', ,LP lobt das Kind', ,LP erteilt eine Zurechtweisung', ,LP droht mit einer Sanktion', ,LP verfügt eine Massnahmec, ,LP verlegt den Arbeitsplatz des Kindes') darauf festgehalten. Pro Minute konnten maximal vier verschiedene Verhaltenskategorien der Kinder und die darauffolgende Reaktion kodiert werden. Pro Verhalten des Kindes wurde eine Lehrpersonenreaktion kodiert. Die Inter-Rater-Reliabilität (Cohens $K$ ) dieser Beobachtungen liegen zwischen .67 und .99 $(M=.80 ; S D=.11)$, was als eine gute bis fast vollkommene Übereinstimmung erachtet wird (Bortz \& Döring, 2005). Bei den Auswertungen wurde zwischen nonverbalen (z. B. Blickkontakt, positive Zurechtweisung) und individualisierenden (z. B. neuer Arbeitsauftrag oder neuer Arbeitsplatz) förderlichen Reaktionen unterschieden. Diese Reaktionen der Lehrperson auf bestimmte Verhaltensweisen wurden über die Lektion addiert und am Total aller Reaktionen gewichtet.

\subsubsection{Weiterbildungsevaluation}

Nach der letzten Schulung bewerteten die Lehrpersonen der Experimentalgruppen mittels Fragebogen unter anderem die einzelnen Elemente des FOKUS-Ansatzes ( 1 , gar nicht hilfreich' bis 6 , ausserordentlich hilfreich').

\subsubsection{Lehrpersonenfragebogen}

Im Onlinelehrpersonenfragebogen wurden unter anderen nachfolgende Konstrukte erhoben:

Mittels des Items „Haben Sie für dieses Schuljahr die Klassenregeln verändert und/oder ergänzt?» mit dichotomem Antwortformat wurden die Änderungen oder Anpassungen der Klassenregeln erhoben. Zur Erhebung der Strukturierung bestimmter Unterrichtsabläufe wurden fünf selbstkonstruierte Aussagen (z. B. „Jeden Morgen erkläre ich vor dem Unterrichtsbeginn den Plan des ganzen Tages anhand einer immer gleichen Darstellungsart.") eingesetzt, welche die Lehrpersonen mit ,trifft zu' oder , trifft nicht zu' beantworteten. Dabei wurden die Anzahl, trifft zu'-Antworten aufsummiert. Die Wertschätzung individueller Bedürfnisse der Kinder wurden auf einer 6er Skala ( 1 ,stimmt überhaupt nicht ' bis 6 ,stimmt voll und ganz') mittels sechs Items (z. B. „Ich gehe oft auf die Gefühle und Empfindungen der Kinder ein.") erhoben, welche zu einem Faktor zusammengefasst wurden ( $\alpha=$ .74). In Anlehnung an die American Psychiatric Association (APA, 2013) schätzte 
die Lehrperson das Verhalten des Kindes in Bezug auf die Unaufmerksamkeit (neun Items z. B. „Es kommt regelmässig vor, dass das Kind nur schwer seine Aufmerksamkeit aufrechterhalten kann.“; $\alpha=.81$ ) und auf die Hyperaktivität/Impulsivität (neun Items z. B. „Es kommt regelmässig vor, dass das Kind mit Händen und Füssen zappelt oder sich auf dem Stuhl windet.“; $\alpha=.92)$ auf einer 6er Skala ( 1 , stimmt überhaupt nicht ' bis 6 , stimmt voll und ganz') ein. Mit drei Items (z. B. „Das Kind ist bei anderen Kindern beliebt.") wurde in Anlehnung an Goodman (1997) die soziale Integration des Kindes im Klassenverband erhoben. Die Interne Konsistenz betrug $\alpha=.81$.

\section{$4 \quad$ Ergebnisse}

Die Wirkung der Weiterbildung wurde summativ überprüft. Es wurden die Mittelwerte zwischen den Gruppen und/oder zwischen Pre- und Posttest auf signifikante Unterschiede untersucht. Aufgrund der geringen Stichprobengrösse sind die inferenzstatistischen Berechnungen mit den Signifikanzniveaus 10\% und 5\% berechnet worden.

Damit eine Weiterbildung die erwartete Wirkung zeigen kann, ist es wichtig, dass Lehrpersonen die Inhalte dieser Weiterbildung im Unterricht umsetzen. Die Lehrpersonen beurteilen die Inhalte der FOKUS-Weiterbildung als, eher hilfreich ' bis , hilfreich'. Die Lehrpersonen, welche eine Weiterbildung besucht haben, haben die Kursinhalte in ihrem Unterricht eingeführt und umgesetzt.

\subsection{Wirkung auf das Verhalten der Lehrpersonen}

Bezüglich der Klassenführung zeigt sich, dass die Lehrpersonen nach der Weiterbildung im Vergleich zur Kontrollgruppe ihre Klassenregeln geändert oder angepasst haben: $\mathrm{X}^{2}(2,130)=22.1, p<.001$, Cramer's $V=.40$. Zudem strukturieren Lehrpersonen der Gruppen A und AB (Weiterbildungsgruppen) im Posttest signifikant mehr Unterrichtsabläufe als im Pretest und im Vergleich zu Lehrpersonen der Kontrollgruppe (Abbildung 3). Die Befunde zeigen zwischen Pre- und Posttest einen signifikanten Anstieg der Anzahl ritualisierter Abläufe im Unterricht auf: $F(1,123)=90.81, p<.01, \eta^{2}=.43$. Aufgrund der Varianzanalyse kann auch eine signifikante Wechselwirkung zwischen dem Messzeitpunkt und der Gruppenzugehörigkeit festgestellt werden: $F(2,123)=4.40, p<.05$. Dies deutet darauf hin, dass beim zweiten Messzeitpunkt nicht nur mehr Unterrichtsabläufe ritualisiert worden 
sind, sondern dass in den Weiterbildungsgruppen die Zunahme der Ritualisierung signifikant ausgeprägter ist als in der Kontrollgruppe.

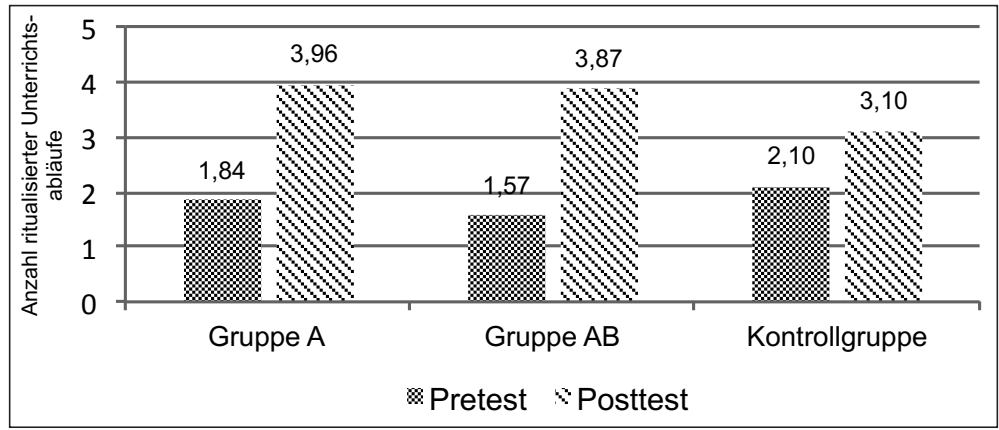

Abb. 3 Anzahl (1 bis 5) ritualisierter Unterrichtsabläufe (Varianzanalysen mit Messwiederholung)

Die Befunde zur berichteten Wertschätzung gegenüber den verhaltensauffälligen und unaufmerksamen Schülerinnen und Schülern (Abbildung 4) zeigen zwischen Pre- und Posttest bei den Weiterbildungsgruppen (A und AB) einen signifikanten Anstieg der Wertschätzung: $F(1,123)=6.18, p<.05, \eta^{2}=.05$. Die Varianzanalyse ergibt zudem einen signifikanten Interaktionseffekt zwischen der Weiterbildungsgruppe und dem Messzeitpunkt: $F(2,123)=3.04, p<.05, \eta^{2}=.05$. Obwohl die Effekte klein sind, bedeutet dies dennoch, dass in den Gruppen $\mathrm{A}$ und $\mathrm{AB}$ die Wertschätzung im Vergleich zur Kontrollgruppe gestiegen ist.

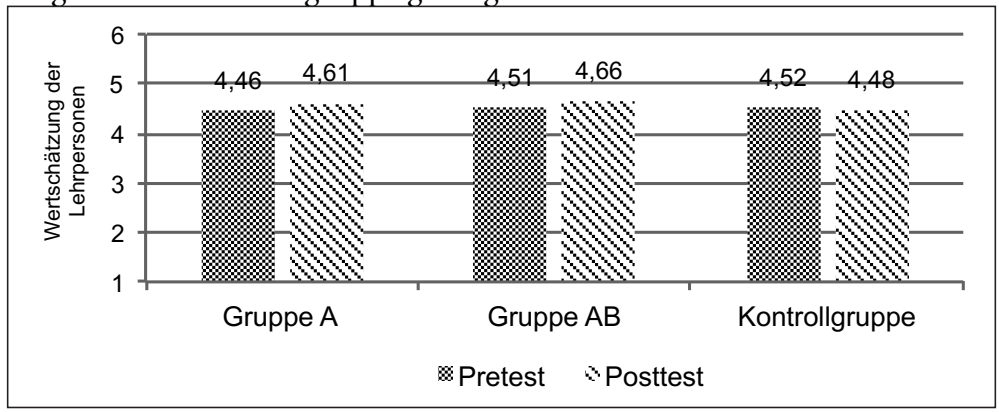

Abb. 4 Wertschätzung (Ausprägungsskala 1 bis 6) gegenüber verhaltensauffälligen und unaufmerksamen Schülerinnen und Schülern (Varianzanalysen mit Messwiederholung) 
Bei den dargestellten Ergebnissen wird der Schwerpunkt auf förderliche Reaktionen der Lehrpersonen gelegt. Anhand der in der Grafik dargestellten Daten (Abbildung 5) ist ersichtlich, dass der Anteil nonverbaler förderlicher Reaktionen auf Hyperaktivität/Impulsivität der Lehrpersonen in den Gruppen A und AB im Vergleich zum Pretest im Posttest grösser ist, wenn auch nicht signifikant: $F(1,115)=1.99$, n.s., $\eta^{2}=.02$. Jedoch unterscheidet sich die Veränderung des Anteiles förderlicher nonverbaler Reaktionen auf hyperaktive/impulsive Verhaltensweisen bei den Weiterbildungsgruppen im Vergleich zur Kontrollgruppe signifikant. Der relative Anteil nonverbaler förderlicher Reaktionen an allen beobachteten Reaktionen bei den Lehrpersonen der Gruppen A und AB ist im zweiten Messzeitpunkt grösser als jener der Kontrollgruppe, welcher in der Tendenz eher abgenommen hat: $F(2,69)=$ 3.96, $p<.05, \eta^{2}=.10$. Im Vergleich dazu weisen die Ergebnisse bezüglich nonverbaler förderlicher Reaktionen auf Unaufmerksamkeit zwischen den Weiterbildungsgruppen und der Kontrollgruppe weder im Zeitvergleich: $F(1,115)=1.99$, n.s., $\eta^{2}=.02$, noch in der Wechselwirkung zwischen Zeit und Gruppenzugehörigkeit: $F(2,123)=$ .29 , n.s., $\eta^{2}=.01$ auf signifikante Veränderungen hin.

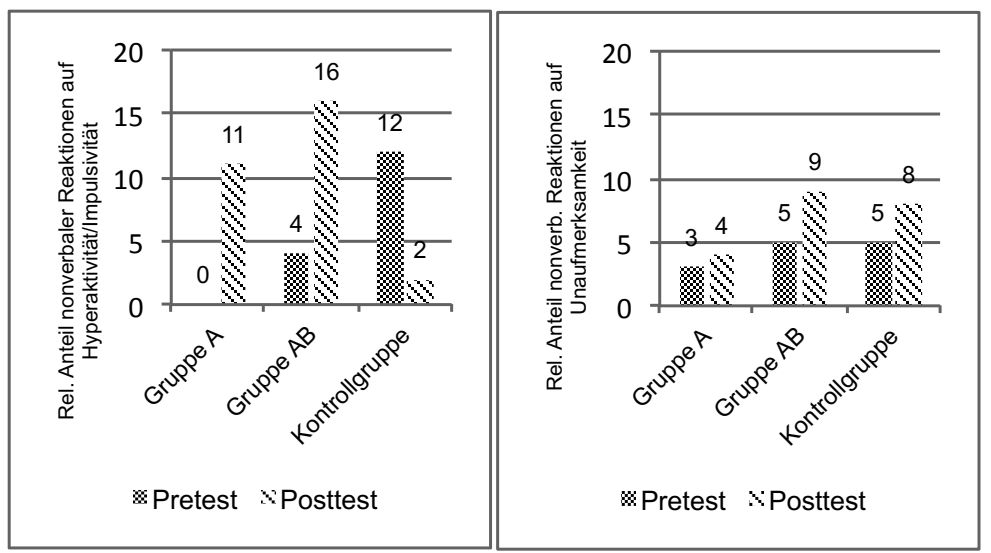

Abb. 5 Anteil beobachteter, nonverbaler förderlicher Reaktionen der Lehrperson auf Unaufmerksamkeit und Hyperaktivität/Impulsivität (Varianzanalysen mit Messwiederholung)

In Abbildung 6 sind die Auswertungen für individualisierende förderliche Reaktionen der Lehrpersonen auf hyperaktive/impulsive und unaufmerksame Verhaltensweisen dargestellt. Anhand der Daten ist ersichtlich, dass Lehrpersonen Hyperaktivität/ 
Impulsivität und Unaufmerksamkeit seltener mit individualisierenden Reaktionen als mit nonverbalen Reaktionen begegnen. Der Vergleich zwischen Pretest und Postest weist auf eine Zunahme förderlicher Reaktionen auf Unaufmerksamkeit hin, welche jedoch nicht signifikant ausfällt: $F(1,115)=.02$, n.s., $\eta^{2}=.00$. Beim Vergleich der Gruppen ist ersichtlich, dass bei den Weiterbildungsgruppen A und $\mathrm{AB}$ der Anteil individualisierender förderlicher Reaktionen auf Unaufmerksamkeit im Vergleich zur Kontrollgruppe, bei welcher dieser Anteil eher abgenommen hat, gestiegen ist. Der Interaktionseffekt ist für Unaufmerksamkeit signifikant: $F(2$, $115)=3.5, p<.05, \eta^{2}=.06$. Lehrpersonen der Weiterbildungsgruppen reagieren öfters als Lehrpersonen der Kontrollgruppe mit individualisierenden förderlichen Reaktionen. Was hingegen die individualisierenden Reaktionen auf Hyperaktivität/ Impulsivität betrifft, wurden weder im Zeitvergleich: $F(1,69)=.42$, n.s., $\eta^{2}=.01$, noch im Interaktionseffekt: $F(2,69)=1.38$, n.s., $\eta^{2}=.04$ signifikante Unterschiede gefunden.

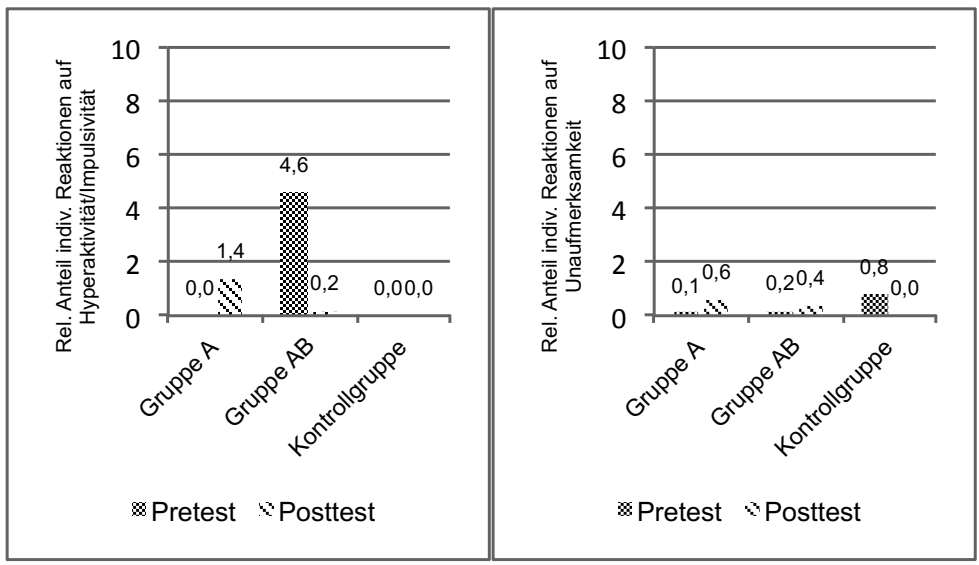

Abb. 6 Anteil beobachteter nonverbaler förderlicher Reaktionen der Lehrperson auf Unaufmerksamkeit und Hyperaktivität/Impulsivität (Varianzanalysen mit Messwiederholung)

\subsection{Wirkung auf das Verhalten des Zielkindes}

Bezüglich der Situation des Zielkindes in der Klasse, gemessen an der von der Lehrperson eingeschätzten sozialen Integration im Klassenverband, weist der Vergleich zwischen Pre- und Posttest auf eine tendenziell erwünschte Entwicklung 
hin (Abbildung 7). Die Veränderung ist nicht signifikant: $F(1,123)=.43$, n.s., $\mathrm{\eta}^{2}=$ .00. Die Varianzanalyse mit Messwiederholung weist aber bezüglich der sozialen Integration eine signifikante Wechselwirkung: $F(2,123)=3.16, p<.05, \eta^{2}=.05$, zwischen dem Messzeitpunkt und der Gruppenzugehörigkeit auf. Kinder, deren Lehrpersonen eine Weiterbildung besucht haben, sind bei der zweiten Messung sozial integrierter in ihrer Klasse als Kinder, deren Lehrperson keine Weiterbildung besucht hat.

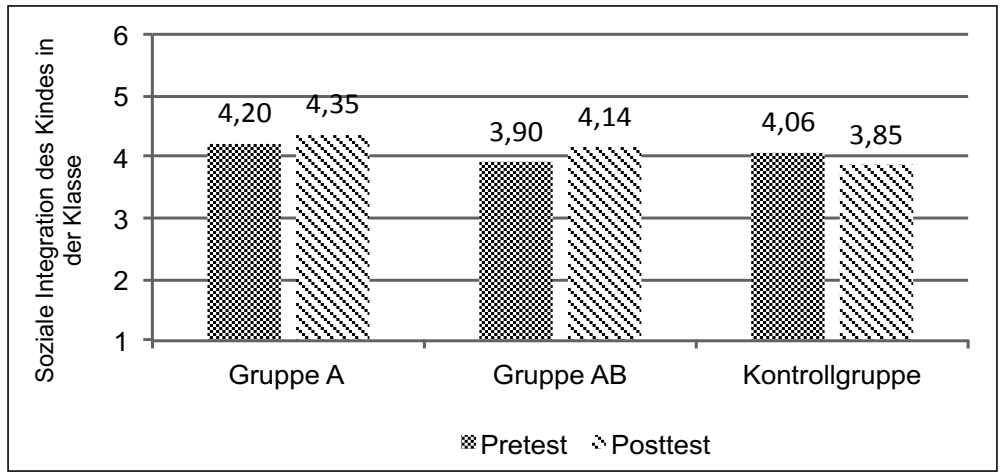

Abb. 7 Einschätzung (Ausprägung der Skala 1 bis 6) der Lehrpersonen zur sozialen Integration des Kindes in der Klasse (Varianzanalysen mit Messwiederholung)

Zudem wurde geprüft, ob sich die Verhaltensweisen der Kinder im Unterricht aufgrund der Weiterbildung positiv verändert haben (Abbildung 8). Anhand der Einschätzung der Lehrpersonen hat sowohl die Hyperaktivität/Impulsivität als auch die Unaufmerksamkeit während der Intervention gesamthaft, d. h. in allen drei Gruppen, abgenommen. Für beide Dimensionen wurden signifikante Veränderungen zwischen dem ersten und zweiten Messzeitpunkt gefunden: Hyperaktivität/Impulsivität: $F(1$, $123)=40.65, p<0.01, \eta^{2}=.25$ und Unaufmerksamkeit: $F(1,123)=109.28, p<0.01, \eta^{2}=.47$. 

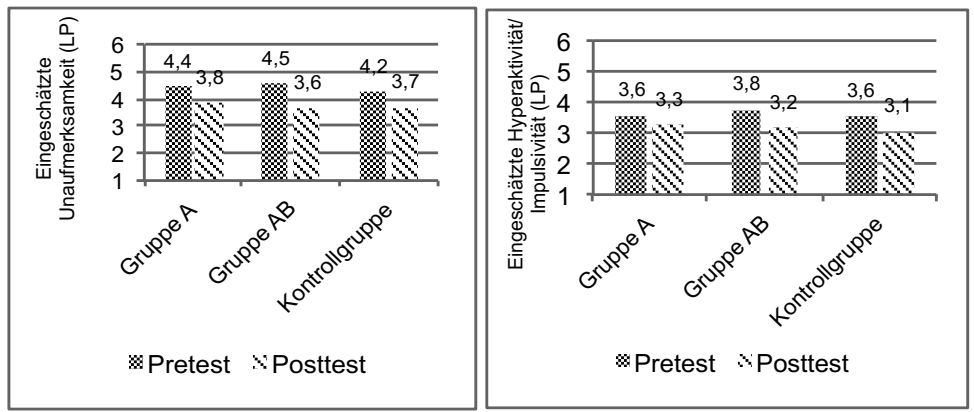

Abb. 8 Einschätzung (Ausprägung der Skala 1 bis 6) seitens der Lehrperson der Hyperaktivität/Impulsivität und Unaufmerksamkeit des Zielkindes während des letzten Monats vor der Erhebung (Varianzanalysen mit Messwiederholung)

Für Unaufmerksamkeit ergibt sich auf dem Zehn-Prozent-Niveau ein signifikanter Interaktionseffekt: $F(2,123)=2.74, p<0.1, \eta^{2}=.04$. Werden die einzelne Weiterbildungsgruppen mit der Kontrollgruppe verglichen, dann ergibt sich ein signifikanter Interaktionseffekt zwischen der Gruppe AB und der Kontrollgruppe: $F(1,74)=4.16$, $p<0.05, \eta^{2}=.05$. Bei den Kindern, deren Lehrpersonen die Weiterbildung zu allen drei Elementen des FOKUS-Ansatzes besucht haben, hat die Unaufmerksamkeit stärker abgenommen als in der Kontrollgruppe. Obwohl die Daten zur Hyperaktivität/Impulsivität eine ähnliche Tendenz aufzeigen, konnte keine signifikante Interaktion ermittelt werden (Tabelle 1). 
Tab. 1 Gruppenvergleich der Einschätzung seitens der Lehrperson der Hyperaktivität/ Impulsivität und Unaufmerksamkeit des Zielkindes während des letzten Monats vor der Erhebung (Varianzanalysen mit Messwiederholung)

\begin{tabular}{|c|c|c|c|}
\hline & Gruppe A & Gruppe AB & Kontrollgruppe \\
\hline \multicolumn{4}{|l|}{ Unaufmerksamkeit } \\
\hline Unaufmerksamkeit $t_{1}$ & $4.43(.70)$ & - & $4.23(.87)$ \\
\hline Unaufmerksamkeit $t_{2}$ & $3.83(.86)$ & - & $3.70(.96)$ \\
\hline $\mathrm{F}, \mathrm{df}_{1}, \mathrm{df}_{2}, \mathrm{p}, \mathrm{R}^{2}$ & $\begin{array}{l}Z: 68.92^{* * *}, 1,87, \\
\eta^{2}=.44\end{array}$ & $\begin{array}{l}G: 1.01,1,87, \\
\eta^{2}=.01\end{array}$ & $\begin{array}{l}\text { I: . } 31,1,87, \\
\eta^{2}=.00\end{array}$ \\
\hline Unaufmerksamkeit $t_{1}$ & - & $4.53(.72)$ & $4.23(.87)$ \\
\hline Unaufmerksamkeit $t_{2}$ & - & $3.64(1.05)$ & $3.70(.96)$ \\
\hline $\mathrm{F}, \mathrm{df}_{1}, \mathrm{df}_{2}, \mathrm{p}, \mathrm{R}^{2}$ & $\begin{array}{l}Z: 63.13^{* * *}, 1,74 \\
\eta^{2}=.46\end{array}$ & $\begin{array}{l}\text { G: } .45,1,74, \\
\eta^{2}=.01\end{array}$ & $\begin{array}{l}\text { I: } 4.16^{*}, 1,74, \\
\eta^{2}=.05\end{array}$ \\
\hline \multicolumn{4}{|c|}{ Hyperaktivität/Impulsivität } \\
\hline Hyperaktivität $\mathrm{t}_{1}$ & $3.58(1.29)$ & - & $3.55(1.29)$ \\
\hline Hyperaktivität $t_{2}$ & $3.26(1.25)$ & - & $3.05(1.29)$ \\
\hline $\mathrm{F}, \mathrm{df}_{1}, \mathrm{df}_{2}, \mathrm{p}, \mathrm{R}^{2}$ & $\begin{array}{l}Z: 29.08^{\star * *}, 1,87, \\
\eta^{2}=.25\end{array}$ & $\begin{array}{l}\text { G: } .23,1,87, \\
\eta^{2}=.00\end{array}$ & $\begin{array}{l}\text { I: } 1.45,1,87, \\
\eta^{2}=.02\end{array}$ \\
\hline Hyperaktivität $\mathrm{t}_{1}$ & - & $3.76(1.05)$ & $3.55(1.29)$ \\
\hline Hyperaktivität $t_{2}$ & - & $3.16(1.13)$ & $3.05(1.29)$ \\
\hline $\mathrm{F}, \mathrm{df}_{1}, \mathrm{df}_{2}, \mathrm{p}, \mathrm{R}^{2}$ & $\begin{array}{l}\mathrm{Z}: 28.47^{\star * *}, 1,74, \\
\eta^{2}=.28\end{array}$ & $\begin{array}{l}\text { G: } .41,1,74 \\
\eta^{2}=.01\end{array}$ & $\begin{array}{l}\text { I: } .25,1,74, \\
\eta^{2}=.00\end{array}$ \\
\hline
\end{tabular}

Legende. ${ }^{* *}: \mathrm{p}<.001,{ }^{* *}: \mathrm{p}<.01,{ }^{*}: \mathrm{p}<.05,+: \mathrm{p}<.10, \mathrm{G}$ : Gruppeneffekt, Z: Zeiteffekt, I: Interaktionseffekt Gruppe mal Zeit, $t_{1}$ : Pretest, $t_{2}$ : Posttest, Gruppe A: verkürzte WB, Gruppe $\mathrm{AB}$ : gesamte WB

\section{$5 \quad$ Schlussfolgerungen}

Hyperaktive/impulsive und unaufmerksame Verhaltensweisen im Unterricht können sich negativ auf die schulische Entwicklung der Kinder auswirken (Döpfner, Frölich \& Lehmkuhl, 2013; Barkley, 2006; Döpfner, 2001). Es gibt aber besondere Unterrichtsgestaltungen und Organisationsformen, von denen hyperaktive/impulsive und unaufmerksame Schülerinnen und Schüler profitieren (Frölich et al., 2014; Zentall, 1993; Gawrilow, Guderjahn \& Gold, 2013). Aus diesem Grund wurde ein Konzept entwickelt, wie verhaltensauffällige und unaufmerksame Schülerinnen und Schüler von den Lehrpersonen der Unterstufe angemessen und längerfristig gefördert werden können. Es wurde geprüft, ob die entwickelte Intervention, die FOKUS-Lehrpersonenweiterbildung, einen positiven Effekt im Hinblick auf die 
effektive Weiterbildung der Lehrpersonen und auf die Veränderung des Umgangs der Lehrpersonen mit verhaltensauffälligen Kindern erzielte. Weiter wurde untersucht, ob die FOKUS-Weiterbildung auf die Vorbeugung oder Reduzierung von Unaufmerksamkeit und impulsiven und hyperaktiven Verhaltensweisen von Kindern des zweiten Schuljahres einen Effekt hatte.

Gesamthaft zeigen die Ergebnisse, dass die Weiterbildung bei den Lehrpersonen auf Akzeptanz gestossen ist und die Lehrpersonen die meisten der vermittelten pädagogischen Strategien angewendet und umgesetzt haben. So nutzten sie beispielsweise die Formulare und Materialien für den Unterricht, die ihnen in der Weiterbildung gezeigt worden sind. Sie wenden aber auch die konkreten Hinweise zur Klassenführung, zur Ritualisierung des Unterrichts sowie zur Klassenzimmergestaltung an. Die erzielten Ergebnisse zeigen, dass Lehrpersonen der Unterstufe durch sehr konkret und direkt auf Anwendungen ausgerichtete Weiterbildungsinhalte erfolgreich, d.h. effektiv, weitergebildet werden können. Bereits in der Weiterbildungssituation wurden im Sinne des situierten Lernens (Mandl, 2004) Anwendungen diskutiert. Dadurch wurde die Verwendbarkeit des vermittelten Wissens und der Hilfsmittel im eigenen Unterricht vereinfacht.

Aufgrund der FOKUS-Weiterbildung haben die Lehrpersonen ihr Verhalten im Unterrichtsalltag verändert. Die Lehrpersonen der Weiterbildungsgruppen strukturieren die Lernumgebung und die Lehrtätigkeit angemessener zur Förderung von verhaltensauffälligen und unaufmerksamen Kindern in der Klasse. Sie achten auf wichtige Aspekte bei der Einführung von Klassenregeln und involvieren die Kinder stärker in den Erarbeitungsprozess derselben. Lehrpersonen, die eine Weiterbildung besucht haben, ritualisieren mehr Unterrichtsabläufe. Dadurch entstehen geeignetere und unterstützendere Lernbedingungen für verhaltensauffällige und unaufmerksame Kinder und die gesamte Klasse.

Die Weiterbildung wirkt sich nicht nur auf die Strukturierung des Unterrichts aus, sondern auch auf die Einstellung der Lehrpersonen gegenüber den auffälligen und unaufmerksamen Schülerinnen und Schülern und auf deren Umgang mit störendem Verhalten. Die Ergebnisse zeigen bezüglich der Wertschätzung der Lehrpersonen gegenüber auffälligen Kindern und ihrer Reaktionsweisen beim Auftreten von störenden Verhaltensweisen positive Veränderungen. Diese treten in der Lehrpersoneneinschätzung auf, werden aber auch in den Verhaltensbeobachtungen sichtbar. Lehrpersonen, die eine Weiterbildung besucht haben, reagieren bei Auftreten von Unaufmerksamkeit oder Hyperaktivität/Impulsivität anteilmässig häufiger mit förderlichen Reaktionen als Lehrpersonen der Kontrollgruppe, wo der Anteil positiver Reaktionen tendenziell abnimmt. Oftmals fallen Lehrpersonen meistens unabsichtlich - in eine negative Reaktionsspirale. Dabei folgen auf störende Verhaltensweisen negative und frustrierte Reaktionen der Lehrpersonen. Diese 
lösen wiederum Frustration beim Kind aus. Durch Letztere werden hyperaktive/ impulsive sowie unaufmerksame Verhaltensweisen verstärkt. Lehrpersonen der Weiterbildungsgruppen haben den Wechsel von der frustrierten, negativen Reaktion gegenüber dem Kind zu einer wertschätzenden, förderlichen Reaktion geschafft. Wird die negative Reaktionsspirale durchbrochen, nimmt auch die Häufigkeit von belastenden Situationen im Unterrichtsalltag ab.

Nebst auf das Verhalten der Lehrpersonen zeigt die Weiterbildung auch eine positive Wirkung a) auf die Situation der Kinder in der Klasse und b) ansatzweise auf ihr Verhalten im Unterricht. Aufgrund der Weiterbildung konnte die soziale Integration des Zielkindes in den Klassenverband im Vergleich zur Kontrollgruppe deutlich verbessert werden. Dabei handelt sich um einen wichtigen Effekt für die Förderung dieser Schülerinnen und Schüler in der Schule. Aus früheren Studien weiss man, dass verhaltensauffällige und unaufmerksame Kinder in der Klasse sozial weniger vorgezogen werden und grössere Schwierigkeiten haben, Freundschaften zu knüpfen und dauerhaft aufrechtzuerhalten (Hoza et al., 2005).

Die Ergebnisse belegen ferner eine positive Wirkung der Massnahmen auf die Unaufmerksamkeit und ansatzweise auf die Hyperaktivität/Impulsivität der Zielkinder. Auffällige und unaufmerksame Verhaltensweisen haben zwischen den beiden Messzeitpunkten abgenommen. Allerdings konnte nur bei den Lehrpersonen, welche die vollständige Weiterbildung besucht haben,nämlich zu allen drei Elementen des FOKUS-Ansatzes (Gruppe AB), eine Abnahme der Unaufmerksamkeit im Vergleich zur Kontrollgruppe belegt werden. Daraus lässt sich schliessen, dass für eine geeignete Förderung von verhaltensauffälligen und unaufmerksamen Kindern in der Klasse das umfassendere Weiterbildungskonzept erfolgreicher ist. Nebst einer angemessenen Klassenführung hat sich die Einführung gezielter kindspezifischer Fördermassnahmen in den Unterrichtsalltag bewährt.

Die Weiterbildungsinhalte und die Evaluationsergebnisse illustrieren, wie durch Lehrpersonenweiterbildung und mit pädagogischen Massnahmen die Situation von unaufmerksamen und hyperaktiven/impulsiven Kindern im Regelunterricht der Grundschule verbessert werden kann. Wegen der kleinen Stichprobe werden nur starke Effekte signifikant. Dadurch wird die Aussagekraft der Befunde zusätzlich erhöht. Die Ergebnisse belegen das grosse Potenzial des FOKUS-Ansatzes für die Bearbeitung von Unaufmerksamkeit und Hyperaktivität/Impulsivität bei Kindern auf der Unterstufe und für die Förderung deren sozialen Einbindung in die Klasse. Insbesondere dieser letzte Aspekt wird von den Lehrpersonen als sehr positiv zurückgemeldet. Durch gezielte pädagogische Interventionen, welche in der Schule von den Lehrpersonen umgesetzt werden können, kann davon ausgegangen werden, dass indirekt eine gewisse Sekundärprävention von Störungen und erschwerten Schulverläufen im Jugend- und Erwachsenenalter geleistet wird. 
Insgesamt findet man eine stärkere Wirkung der Weiterbildung auf Unaufmerksamkeit als auf Hyperaktivität/Impulsivität. Die Ergebnisse zeigen generell, dass sich die Bedingungen von Unaufmerksamkeit von den Bedingungen der Hyperaktivität/ Impulsivität unterscheiden. Entsprechend müssen zukünftig für beide Verhaltensweisen spezifische Massnahmen entwickelt und umgesetzt werden. In Bezug auf die Bearbeitung der Hyperaktivität/Impulsivität mit Hilfe des FOKUS-Ansatzes gibt es einen gewissen Optimierungsbedarf bei der Weiterbildung. Es müssen zukünftig weitere Strategien mit nachweisbarer Wirkung beim Umgang mit hyperaktiven/ impulsiven Kindern im Regelunterricht erarbeitet werden. Diese Erkenntnisse sind wichtig, um zukünftigen Analysen der Wirkung der FOKUS-Weiterbildung auch auf anderen Schulstufen (Kindergarten oder höhere Klassen der Primarschule) erfolgreich replizieren zu können.

\section{Literatur}

American Psychiatric Association (2013). Diagnostic and statistical manual of mental disorders: DSM-5. American Psychiatric Association (5. Auflage). Arlington, Va.: American Psychiatric Association.

Bader, M., Pierrehumbert, B., Junier, L., \& Halfon, O. (2005). Die Aufmerksamkeitsdefizit-Hyperaktivitäts-Störung bei Kindern und Jugendlichen. Bericht über eine 2001 in Morges durchgeführte Studie und eine Umfrage bei der Ärzteschaft des Kantons Waadt. Lausanne: BAG.

Barkley, R. A. (2006). Attention Deficit-Hyperactivity Disorder: A handbook for diagnosis and treatment (3. Aufl.). New York: Guilford.

Barkley, R. A., Fischer, M., Edelbrock, C., \& Smallish, L. (1991). The adolescent outcome of hyperactive children diagnosed by research criteria: III. Mother-child interactions, family conflicts and maternal psychopathology. Journal of Child Psychology and Psychiatry, 32, 233-256.

Bortz, J. \& Döring, N. (2007). Forschungsmethoden und Evaluation für Human-und Sozialwissenschaftler: Limitierte Sonderausgabe. Springer-Verlag.

Döpfner, M. (2001). Hyperaktivität und Impulsivität. In D. h. Rost (Hrsg.), Handwörterbuch pädagogische Psychologie (S. 260-267). Weinheim: Beltz PVU.

Döpfner, M., Schürman, S., \& Frölich, J. (2002). Therapieprogramm für Kinder mit hyperkinetischem und oppositionellem Problemverhalten THOP. Weinheim: Beltz.

Döpfner, M., Frölich, J., \& Lehmkuhl, G. (2013). Aufmerksamkeitsdefizit-/Hyperaktivitätsstörung (ADHS). Göttingen: Hogrefe.

DuPaul, G.J., \& Stoner, G. (2003). ADHD in the schools. New York: Guilford.

DuPaul, G.J., Weyandt, L.L., \& Janusis, G.M. (2011). ADHD in the classroom: Effective interventions strategies. Theorie into practice, 50(1), 35-42. 
Frölich, J., Döpfner, M., \& Banaschewski, T. (2014). ADHS in Schule und Unterricht: Pädagogisch-didaktische Ansätze im Rahmen des multimodalen Behandlungskonzepts. Stuttgart: Kohlhaammer.

Gawrilow, C., Guderjahn, L., \& Gold, A. (2013). ADHS: Schluss mit Chaos im Klassenzimmer. München: Reinhardt.

Gawrilow, C., Gollwitzer, P. M., \& Oettingen, G. (2011). If-then plans benefit delay of gratification performance in children with and without ADHD. Cognitive Therapy Research, 35, 442-455.

Gawrilow, C., Schmitt, K., \& Rauch, W. (2011). Kognitive Kontrolle und Selbstregulation bei Kindern mit ADHS. Kindheit und Entwicklung, 20(1), 41-48.

Heim, D., \& Nido, M. (2008). Burnout im Lehrberuf. Aarau: FHNW.

Hoberg, K. (2013). Schulratgeber ADHS. Ein Leitfaden für LehrerInnen. München: Ernst Reinhardt.

Hoza, B., Gerdes, A.C., Mrug, S., Hinshaw, S. P., Bukowski, W. M., Gold, J.A., Arnold, L. E., Abikoff, H. B., Conners, C. K., Elliott, G. R., Greenhill. L L., Hechtman, L., Jensen, P. S., Kraemer, H. C., March, J. S., Newcorn, J. H., Severe, J. B., Seanson, J. M., Vitiello, B., Wells, K. C., \& Wigal, T. (2005). Peer-assessed Outcomes in the Multimodal Treatment Study of Children with Attention-Deficit/Hyperactivity Disorder. Journal of Clinical Child and Adolescent Psychology, 34, 74-86.

Jarman, F. C. (1996). Current approaches to management of attention deficit hyperactivity disorder. The Australian Educational and Developmental Psychologist, 13, 46-55.

Kos, J. M., Richdale, A. L., \& Hay, D. A. (2006). Children with attention deficit hyperactivity disorder and their teachers: A review of the literature. International Journal of Disability, Development and Education, 53, 147-160.

Lidzba, K., Christiansen, H., \& Drechsler, R. (2013). Conners 3 (R). Conners Skalen zu Aufmerksamkeit und Verhalten - 3 (Deutschsprachige Adaptation der Conners 3rd Edition (R) (Conners $3(R)$ von C. Keith Conners). Bern: Huber.

Mandl, H. (2004). Gestaltung problemorientierter Lernumgebungen. Journal für Lehrerinnen- und Lehrerbildung, 4(3), 47-51.

Mannuzza, S., Klein, R.G., Bessler, A., Malloy P., \& Hynes, M. E. (1997). Educational and occupational outcome of hyperactive boys grown up. Journal of the American Academy of Child \& Adolescent Psychiatry, 36(9), 1222-1227.

Maslach, C., \& Jackson, S. (1981). The measurement of experienced burnout. Journal of Occupational Behavior, 2, 99-113.

Mautone, J.A., Lefler, E.K., \& Power, T.J. (2011). Promoting family and school success for children with ADHD: Strengthening relationships while building skills. Theory Into Practice, 50(1), S. 43-51.

Neuenschwander, M. P. \& Benini, S. (2017). Der FOKUS-Ansatz bei verhaltensauffälligen und unaufmerksamen Kindern konkret. Schweizerische Zeitschrift für Heilpädagogik, 23(3), 46-52..

Neuenschwander, M. P. \& Malti, T. (2009). Selektionsprozesse beim Übergang in die Sekundarstufe I und II. Zeitschrift für Erziehungswissenschaft, 12(2), 216-232.

Neuenschwander, M. P., Balmer, T., Gasser, A., Goltz, S., Hirt, U., Ryser, H., \& Wartenweiler, H. (2005). Schule und Familie - was sie zum Schulerfolg beitragen. Bern: Haupt.

Zentall, S. S. (1993). Research on the educational implications of attention deficit hyperactivity disorder. Exceptional Children, 60, 143-153. 
Open Access Dieses Kapitel wird unter der Creative Commons Namensnennung 4.0 International Lizenz (http://creativecommons.org/licenses/by/4.0/deed.de) veröffentlicht, welche die Nutzung, Vervielfältigung, Bearbeitung, Verbreitung und Wiedergabe in jeglichem Medium und Format erlaubt, sofern Sie den/die ursprünglichen Autor(en) und die Quelle ordnungsgemäß nennen, einen Link zur Creative Commons Lizenz beifügen und angeben, ob Änderungen vorgenommen wurden.

Die in diesem Kapitel enthaltenen Bilder und sonstiges Drittmaterial unterliegen ebenfalls der genannten Creative Commons Lizenz, sofern sich aus der Abbildungslegende nichts anderes ergibt. Sofern das betreffende Material nicht unter der genannten Creative Commons Lizenz steht und die betreffende Handlung nicht nach gesetzlichen Vorschriften erlaubt ist, ist für die oben aufgeführten Weiterverwendungen des Materials die Einwilligung des jeweiligen Rechteinhabers einzuholen.

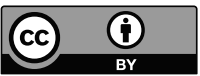

